\title{
Proteomic Analysis of Two Non-Bronchoscopic Methods of Sampling the Lungs of Patients with the Acute Respiratory Distress Syndrome (ARDS)
}

\author{
Dong W. Chang • Giuseppe Colucci • Tomas Vaisar • \\ Trevor King • Shinichi Hayashi • Gustavo Matute-Bello • \\ Roger Bumgarner • Jay Heinecke • Thomas R. Martin • \\ Guido M. Domenighetti
}

Published online: 5 January 2008

(C) Humana Press Inc. 2007
BAL samples, $13.2 \%$ were increased in s-Cath compared to mini-BAL, and $18.4 \%$ were decreased in s-Cath compared to mini-BAL. For each of the seven subjects, overabundance analysis showed that the actual number of differentially expressed spots in the mini-BAL and s-Cath sample was more than the expected number if the samples were identical. There were nine proteins that were consistently differentially expressed between the mini-BAL and s-Cath samples. Of these nine proteins, five are abundantly found in neutrophils or airway epithelial cells, suggesting that the s-Cath may sample the bronchial airways to a greater extent than mini-BAL.

Conclusion Proteomic analysis of mini-BAL and s-Cath samples shows for the first time that, although these two methods for sampling the lungs of critically ill patients are generally similar, the s-Cath method oversamples the distal airways compared to the mini-BAL method.

This study was funded by NIH/NHLBI HL073996.

D. W. Chang $\cdot \mathrm{S}$. Hayashi $\cdot$ G. Matute-Bello $\cdot$ T. R. Martin Medical Research Service of the VA Puget Sound Healthcare System, University of Washington,

Seattle, WA, USA

D. W. Chang $\cdot \mathrm{S}$. Hayashi $\cdot$ G. Matute-Bello $\cdot$ T. R. Martin Divisions of Pulmonary and Critical Care Medicine, University of Washington, Seattle, WA, USA

T. Vaisar $\cdot$ J. Heinecke

Division and Endocrinology and Metabolism, University of Washington, Seattle, WA, USA

T. King $\cdot$ R. Bumgarner Department of Medicine, University of Washington, Seattle, WA, USA

T. King $\cdot$ R. Bumgarner Department of Microbiology,

University of Washington,

Seattle, WA, USA

G. Colucci · G. M. Domenighetti

Multidisciplinary Intensive Care Unit,

Regional Hospital,

Locarno, Switzerland

T. R. Martin $(\triangle)$

Seattle VAMC Pulmonary Research Laboratories,

VA Puget Sound Health Care System,

1660 S. Columbian Way, 151L,

Seattle, WA 98108, USA

e-mail: trmartin@u.washington.edu 
Keywords Acute respiratory distress syndrome . Bronchoalveolar lavage · Bronchoscopy · Lung injury · Proteomics

\section{Introduction}

Studies of the mechanisms of acute lung injury (ALI) and the acute respiratory distress syndrome (ARDS) have relied heavily on analyses of fluid from the distal airspaces of humans and animals with normal and injured lungs. The major methods of sampling the cells, proteins, and other constituents of the airspaces include direct collection of edema fluid from the distal airways using a long suction catheter (s-Cath), the use of a suction catheter with an occlusive tip through which a small volume of fluid is instilled and then aspirated (mini-BAL), and directed fiberoptic bronchoscopy with bronchoalveolar lavage in a specific lung region, such as the right middle lobe or lingula (bronchoscopic-BAL). The s-Cath and the miniBAL methods are advantageous because they are safe, inexpensive, and easy to perform. In contrast, bronchoscopy is more frequently associated with complications such as respiratory deterioration, is more expensive, and requires highly trained personnel. Importantly, relatively little is known about how these methodologies compare in sampling the distal airspaces.

The s-Cath method was developed and validated by Matthay and Wiener-Kronish [1] and has the advantage of recovering undiluted edema fluid that moves up from the alveolar space into the distal airways. Micropuncture studies in animals have shown that this fluid accurately reflects alveolar fluid composition under experimental conditions [2, 3]. However, patients with lung injury typically have recoverable lung edema only at the onset of illness, so this method is not suitable for studying sequential changes over time. The mini-BAL method was developed to enable sampling of the distal airspaces regardless of whether or not edema fluid is present in the airways. In this method, a specialized dual lumen catheter is advanced through the endotracheal tube until it occludes the distal airway, then a small volume of $0.9 \% \mathrm{NaCl}$ is instilled into the distal lung segment and recovered by aspiration. This method can be used to sample the distal airspaces repeatedly, as long as the patient remains intubated.

The essential problem in comparing studies using these two minimally invasive methodologies is that their comparability in recovering airspace cells and proteins has not been investigated. Because the mini-BAL method involves instilling fluid into the lungs, it is possible that the miniBAL method samples a different 'space' than the s-Cath method. Therefore, the purpose of this study was to assess the similarity between the lung fluids obtained by s-Cath and mini-BAL. We performed a proteomic analysis of distal lung fluids obtained by both techniques in patients with ARDS based on the premise that comparing the global protein content in the lung fluids obtained by each method would be a relevant measure of the similarity between these lung fluid samples. Furthermore, examining the characteristics of the proteins that are differentially recovered by each technique could reveal underlying sampling biases.

\section{Materials and Methods}

\section{Patient Population}

Patients admitted to the multidisciplinary intensive care unit at Regional Hospital, Locarno, Switzerland between 2001 and 2003 were eligible for inclusion. All subjects were prospectively identified as having ALI/ARDS by the American-European Consensus Conference definition [4, 6]. Clinical and physiologic indices of disease severity were recorded using a standardized data collection form. The human study protocol was approved by the Ethical Committee of Human Research of Canton Ticino, Switzerland. The use of the lung fluid samples for proteomic analysis was approved by the Institutional Review Board of the University of Washington. Informed consent was obtained from the patient or legal representative.

\section{Sample Collection}

All patients were intubated and mechanically ventilated at the time of sample collection. Distal lung fluid samples were obtained from each subject using both the s-Cath and mini-BAL techniques within $5 \mathrm{~h}$ of intubation. The s-Cath was performed using a 14-Fr tracheal suction catheter. The catheter was blindly advanced through the endotracheal tube until it was wedged into a distal airway. Gentle aspiration without saline instillation was used to collect lung fluid samples as previously described by Matthay and Wiener-Kronish [1]. The mini-BAL samples were collected using a dual lumen catheter (16-Fr $5 \mathrm{~mm}$ outer catheter, 12-F inner catheter, BAL Cath, Ballard Medical Products, Draper, Utah, USA) that was blindly introduced into the airways via the endotracheal tube. The inner catheter was advanced into a distal wedge position, and five separate aliquots of $30 \mathrm{ml}$ of sterile $0.9 \% \mathrm{NaCl}$ were serially instilled and gently suctioned from the distal airways. Mini-BAL samples were obtained by the local investigators, whereas s-Cath samples were obtained by either an investigator or a trained critical care nurse. All samples were immediately processed by an investigator. 
Sample Preparation

All samples were filtered through a $100-\mu \mathrm{m}$ nylon cell strainer (Falcon 2360, Becton Dickinson, Frankling Lakes, NJ, USA). An aliquot of the sample fluid was used for cell counts obtained manually with a hematocytometer. A portion of the cell-free supernatant was frozen at $-80^{\circ} \mathrm{C}$ and sent to the VA Puget Sound Pulmonary Research Laboratories (Seattle, WA, USA) for proteomic studies.

For proteomic analysis, all samples were concentrated to equivalent starting volumes (approximately $500 \mu \mathrm{l}$ ) using a $5 \mathrm{kDa}$ molecular weight filter (Amicon Ultra-15, Millipore, MA, USA). The samples were then spun through a $0.22-\mu \mathrm{m}$ filter to remove mucus and other insoluble products. The total protein concentrations were determined using the BCA Protein Assay (Pierce, Rockford, IL, USA).

To improve the detection of low-abundance proteins in proteomic analysis, all samples were depleted of six highly abundant serum proteins (albumin, transferrin, haptoglobin, antitrypsin, IgG, IgA) using a monoclonal IgG immunoaffinity high-performance liquid chromatography column (Multiple Affinity Removal System, Agilent Technologies, Wilmington, DE, USA). Approximately 1,500 $\mu \mathrm{g}$ of lung fluid protein was applied to the column. The flow-through fractions containing the unbound, low-abundance proteins were collected for further analysis. When lung fluid samples exceeded $1,500 \mu \mathrm{g}$ of total protein, sequential injections onto the column were performed, and the flowthrough fractions were pooled. The high-abundance proteins that were depleted from the lung fluids were eluted from the immunoaffinity column and analyzed using the same methodology as the flow-through fraction containing the low-abundance proteins.

Following the depletion of high-abundance proteins, the buffer of the flow-though fraction was replaced with a buffer containing $7 \mathrm{M}$ urea, $2 \mathrm{M}$ thiourea, $4 \%$ CHAPS, and $10 \mathrm{mM}$ tris at $\mathrm{pH} 8.5$. The flow-through fraction was then concentrated to approximately $100 \mu \mathrm{l}$ using a $5-\mathrm{kDa}$ molecular weight filter (Amicon Ultra-4, Millipore, MA, USA). The final protein concentration was measured using the 2D-Quant Assay (Amersham Biosciences, Piscataway, NJ, USA), which allows protein measurements in ureabased solutions.

\section{Two-Dimensional Difference Gel Electrophoresis}

Proteins in the lung fluid samples were analyzed using twodimensional difference gel electrophoresis (DIGE, Amersham). In the DIGE method, fluorescent dyes with unique absorbance and emission spectra (Cy2, Cy3, Cy5) are used to differentially label a reference standard (a pooled collection of all the samples used in the experiment) and up to two experimental samples. The aliquots of the labeled standard and each labeled experimental sample are then mixed and run in a single two-dimensional gel in which proteins are separated first by their isoelectric points (first dimension) and then by molecular weights (second dimension). The gel is scanned at three different wavelengths to identify the proteins labeled with $\mathrm{Cy} 2, \mathrm{Cy} 3$, and $\mathrm{Cy} 5$, and the images are then superimposed. The abundance of the individual protein spots in the experimental samples are expressed as a ratio between the spot of interest $(\mathrm{Cy} 3$ or $\mathrm{Cy}$ 5 label) and the corresponding spot in the reference standard (Cy2 label). The pooled standard is run with the samples of interest in each gel to minimize the experimental variability associated with gel-to-gel comparisons [5].

For each subject, $50 \mu \mathrm{g}$ of pooled standard, $50 \mu \mathrm{g}$ of fluid from s-Cath, and $50 \mu \mathrm{g}$ of fluid from mini-BAL were labeled using $\mathrm{Cy} 2, \mathrm{Cy} 3$, and $\mathrm{Cy} 5$, respectively, to create a single sample containing a mixture of the three different labeled samples. These samples were then applied onto a single rehydrated immobilized $\mathrm{pH}$ gradient (IPG) strip pH 4-7, $24 \mathrm{~cm}$ (Amersham). For first-dimensional electrophoresis, voltage was applied at $200 \mathrm{~V}$ for $3 \mathrm{~h}$, increased linearly from 300 to $8,000 \mathrm{~V}$ for $12 \mathrm{~h}$, and maintained at $8,000 \mathrm{~V}$ for $4 \mathrm{~h}$. The IPG strip was removed and equilibrated in a buffer containing $100 \mathrm{mM}$ tris $(\mathrm{pH} \mathrm{8.0)}$, $6 \mathrm{M}$ urea, $35 \% v / v$ glycerol, $2 \% \mathrm{w} / v$ sodium dodecyl sulfate (SDS), and $32 \mathrm{mM}$ dithiothreitol (DTT). The strip was then equilibrated a second time with the same solution except that the DTT was replaced with $0.24 \mathrm{M}$ iodoacetamide. The strip was then applied onto a $21 \times 27 \mathrm{~cm}$ low-fluorescence, $12.5 \%$ SDS gel. The gel was run at a power of $5 \mathrm{~W}$ for $30 \mathrm{~min}$, then at $20 \mathrm{~W}$ for approximately $5 \mathrm{~h}$ using the Ettan Dalt Twelve System (Amersham). To identify individual protein spots, the gels were scanned using the GE Healthcare Typhoon 9400 Series Variable Imager with excitation wavelengths of $526 \mathrm{~nm}$ for $\mathrm{Cy} 2,532 \mathrm{~nm}$ for $\mathrm{Cy} 3$, and $580 \mathrm{~nm}$ for Cy5. This procedure was performed using the samples from each of the seven subjects, yielding a total of seven gels, each containing the s-Cath and mini-BAL sample for one patient and the standard sample consisting of a mixture of all proteins in all samples.

\section{Protein Spot Analysis}

Each gel contained spots from the pooled standard (Cy2 wavelength), s-Cath (Cy3), and mini-BAL (Cy5). By scanning the gel at the excitation wavelengths for each fluorescent dye, comparisons between the protein spots in the standard (Cy2) and the samples of interest $(\mathrm{Cy} 3,5)$ were made. The abundance of each of the protein spots in the experimental samples was expressed as a ratio between the spot of interest in the s-Cath and mini-BAL sample (Cy3 or Cy 5 label) and the corresponding spot in the reference standard (Cy2 wavelength). We performed initial studies 
using samples containing known amounts of proteins and found that the lower limit of detection and protein identification in the DIGE method is approximately 50$70 \mathrm{ng}$ (data not shown). Protein spot detection and spot abundance comparisons were performed using the Decyder software program version 5.01 (Amersham). The difference in-gel analysis (DIA) module of the Decyder program was used to detect differences in the abundance of the individual spots between the s-Cath and mini-BAL samples for each subject (Cy3/Cy2 vs $\mathrm{Cy} 5 / \mathrm{Cy} 2$, pairwise comparison). A significant difference in spot abundance was defined as a twofold change in the log (volume) ratio. The Student's $t$ test was used for comparison with a $p<0.05$ for statistical significance. The pairwise comparison was used to measure the number of spots that were differentially expressed between the s-Cath and mini-BAL samples. Following the pairwise comparison, an overabundance analysis was performed for each patient by comparing the actual number of differentially expressed spots detected in the pairwise analysis with the calculated number of differentially expressed spots expected if the mini-BAL and s-Cath samples were equivalent at a significance of $p<0.05$. The expected number of differentially expressed spots, which is an estimate of the number of differentially expressed spots caused by chance alone, was calculated by multiplying the number of spots detected in the mini-BAL and s-Cath sample for each patient by the $p$ value 0.05 . The pairwise comparison and the overabundance analysis were used to measure the degree of similarity between the s-Cath and mini-BAL samples for each subject.

Next, the biological variance analysis module of the Decyder program was used to group protein spots found in mini-BAL samples across the seven subjects and to compare them to those spots found in s-Cath samples from the same subjects (group-wise comparison). Only the spots that were detected in at least six out of seven mini-BAL and s-Cath samples were included in the group-wise comparison. Significant differences in spot abundance were calculated using Student's $t$ test $(p<0.05)$. The group-wise comparison was used to find protein spots that were consistently different between the mini-BAL and s-Cath samples.

\section{Identification of Protein Spots}

Protein spots that were differentially expressed between the mini-BAL and s-Cath samples in the group-wise analysis were excised using an automated spot picker (Ettan DIGE Spot-picker, Amersham) with a 2-mm picking head and placed in a 96-well ZipPlateC18 (Millipore, MA, USA) for in-gel digestion and analysis using matrix-assisted laser desorption mass spectrometry (MALDI). Each gel spot containing a protein of interest was washed, dehydrated, and digested with trypsin in $50 \mu$ of $50 \mathrm{mM} \mathrm{NH}_{4} \mathrm{HCO}_{3}$ and $5 \mathrm{mM} \mathrm{CaCl}_{2}$ (27). The peptides were eluted from the ZipTip of the plate in $80 \%$ acetonitrile containing $0.1 \%$ trifluoroacetic acid (TFA), spotted on the MALDI target $(0.5 \mu \mathrm{l})$, dried, and overlaid with $0.5 \mu \mathrm{l}$ of $\alpha$-cyano-4hydroxycinnamic acid matrix $(5 \mathrm{mg} / \mathrm{ml}$ in $70 \%$ acetonitrile, $0.1 \%$ TFA) for matrix-assisted laser desorption/ionization time-of-flight (MALDI-TOF)/TOF mass spectrometry. The MALDI-TOF MS and MS/MS spectra were acquired on the ABI 4700 proteomic analyzer MALDI tandem TOF mass spectrometer (Applied Science, Foster City, CA, USA) with air as the collision gas. The MS and MS/MS spectra were searched in combination against the human SwissProt protein database using the Mascot search engine. A cutoff of total peptides $>5$ and uniques peptides $>2$ was used for protein identification. Only protein identifications with a confidence interval greater than $95 \%$ were further considered.

\section{Statistical Analysis}

The clinical characteristics of the study subjects are reported as the mean \pm SD. Comparisons between the characteristics of the lung fluid samples were made using an unpaired, twotailed $t$ test. Differences in the abundance of protein spots were determined using the Decyder software program version 5.01 (Amersham) as described above. The proteins in the spots were identified by comparing the spectra obtained from mass spectrometry to the SwissProt protein database. Significance levels were set at a $p<0.05$.

\section{Results}

\section{Patient Characteristics}

The baseline clinical characteristics and the risk factors for ARDS of the seven patients included in the study are summarized in Table 1. The patients were male with a mean

Table 1 Patient characteristics $(N=7)$

\begin{tabular}{ll}
\hline Characteristic & Description \\
\hline Age & $54 \pm 7.8$ \\
$\mathrm{Male} /$ female & $7 / 0$ \\
$\mathrm{PaO}_{2} / \mathrm{FiO}_{2}$ & $143 \pm 37.9$ \\
$\mathrm{Lung}_{\text {injury score (LIS) }}$ & $2.3 \pm 0.5$ \\
$\mathrm{SAPS}$ II & $55 \pm 20.3$ \\
ICU mortality (\%) & $2 / 7(28.6)$ \\
Risk factors for ARDS & \\
Sepsis & $2 / 7$ \\
Pneumonia & $3 / 7$ \\
Pancreatitis & $1 / 7$ \\
Drug toxicity & $1 / 7$ \\
\hline
\end{tabular}


age of 54 years. There were several different risk factors for ARDS, with pneumonia being the most common (three out of seven). The characteristics of the lung fluids obtained by $\mathrm{s}$-Cath and mini-BAL obtained in these patients are summarized in Table 2. The cell count for one of the s-Cath samples could not be obtained because of low sample volume. The s-Cath samples had significantly more white blood cells than the mini-BAL samples $(p<0.05)$. Although the percentage of neutrophils was higher in the $\mathrm{s}$-Cath samples, it did not reach statistical significance ( $p=$ 0.192). In addition, four out of seven the s-Cath samples contained bronchial epithelial cells compared to two out of seven mini-BAL samples. The protein concentration of the s-Cath samples was approximately 16 -fold greater than the mini-BAL samples $(p<0.05)$.

\section{Depletion of High-abundance Proteins}

Before proteomic analysis, the lung fluids obtained by both s-Cath and mini-BAL were depleted of six high-abundance proteins (albumin, anti-trypsin, haptoglobin, transferrin, $\mathrm{IgA}, \mathrm{IgG}$ ) using an immunoaffinity column. Depletion of high-abundance proteins improved the detection of the number of low-abundance protein spots by at least threefold (undepleted vs low-abundance fractions, Fig. 1). The highabundance fraction was analyzed to assess the inadvertent depletion of low-abundance proteins. Nineteen spots in the high-abundance fraction could not be assigned to one of the high-abundance proteins based on their location on the gel. These spots were excised and identified by mass spectrometry. Fifteen of the 19 spots were identified as one of the six highabundance proteins depleted by the immunoaffinity column. Four protein spots in the high-abundance fraction were not among the six proteins that are depleted by the column (Fig. 1).

\section{Spot Analysis}

To determine whether the lung fluid samples collected by the s-Cath and mini-BAL were equivalent, the protein

Table 2 Characteristics of lung fluids obtained by s-Cath and mini-BAL

\begin{tabular}{llll}
\hline Samples & $\begin{array}{l}\text { s-Cath } \\
(N=6)\end{array}$ & $\begin{array}{l}\text { Mini-BAL } \\
(N=7)\end{array}$ & $p$ Value \\
\hline $\begin{array}{llll}\text { White blood cells (per } \\
\quad \text { microliter of sample fluid) }\end{array}$ & $2067 \pm 1193$ & $771 \pm 642$ & 0.047 \\
Neutrophils (\%) & $82 \pm 19$ & $66 \pm 23$ & 0.192 \\
Macrophages (\%) & $14 \pm 18$ & $29 \pm 24$ & 0.227 \\
Lymphocytes (\%) & $2.8 \pm 1.7$ & $3.6 \pm 2.8$ & 0.527 \\
Eosinophils (\%) & $1.0 \pm 1.2$ & $1.0 \pm 1.6$ & 0.443 \\
Epithelial cells & $4 / 7$ & $2 / 7$ & \\
Protein concentration (g/dl) & $25.3 \pm 22.3$ & $1.6 \pm 2.0$ & 0.048 \\
\hline
\end{tabular}

profiles in the fluid obtained by each technique in the same patient were compared using DIGE. On average, 2,164 spots were detected in the spot analysis (Table 3). These spots were used for pairwise comparison of protein abundance in the mini-BAL and s-Cath samples from each subject. The similarity in spot abundance was used as a measure of the agreement between the samples obtained by each method. Figure 3 shows a gel from one representative subject that demonstrates how this comparison was made using DIGE.

The differences in spot abundance between the s-Cath and mini-BAL fluids in each of the seven subjects are summarized in Table 3. On average, the abundance of $68.4 \%( \pm 8.9 \%)$ of the protein spots was equivalent for each subject. In contrast, the abundance of $31.6 \%$ of the protein spots was different in the s-Cath vs mini-BAL samples in each patient. Of these, $13.2 \%( \pm 7.1 \%)$ of the protein spots were increased in s-Cath compared to mini-BAL, and $18.4 \%( \pm 4.8 \%)$ of the proteins spots were decreased in s-Cath compared to mini-BAL. To evaluate the experimental variability in our analysis, identical s-Cath samples were labeled with Cy3 and Cy5 and run on a single gel (Fig. 2). The overlap image in Fig. 2 shows that the location of the protein spots is not affected by whether they are labeled with $\mathrm{Cy} 3$ or Cy5. Furthermore, the spot analysis program (Decyder) correctly identified all 2,257 detected spots as similar in abundance (Fig. 2). In contrast, when s-Cath and mini-BAL were run in the same DIGE analysis, many spots differed (Fig. 3).

The number of differentially expressed spots in the s-Cath and mini-BAL sample was compared with the calculated number of differentially expressed spots expected if the s-Cath and mini-BAL samples were equivalent (overabundance analysis). This analysis showed that the actual number of different spots in the mini-BAL and s-Cath sample was more than the expected number for each of the seven subjects (Fig. 4). On average, there was a sevenfold increase in the actual number of differentially expressed spots as compared with the calculated number. The minimum difference was a fourfold increase (sample 1, Fig. 4), whereas the maximum difference was nearly a 12fold increase (sample 2, Fig. 4).

Because of the overabundance of differentially expressed spots between the s-Cath and mini-BAL samples, the next step was to perform a groupwise comparison to determine whether there were consistent differences in the protein spots found in the s-Cath vs mini-BAL samples. Seven hundred five protein spots were detected in both the mini-BAL and s-Cath fluids of six out of seven subjects and were used for the groupwise comparison (Fig. 5). Of the 705 spots, 17 $(2.4 \%)$ were differentially expressed in s-Cath vs mini-BAL samples. All of the 17 spots were increased in the s-Cath samples as compared with the mini-BAL samples. 


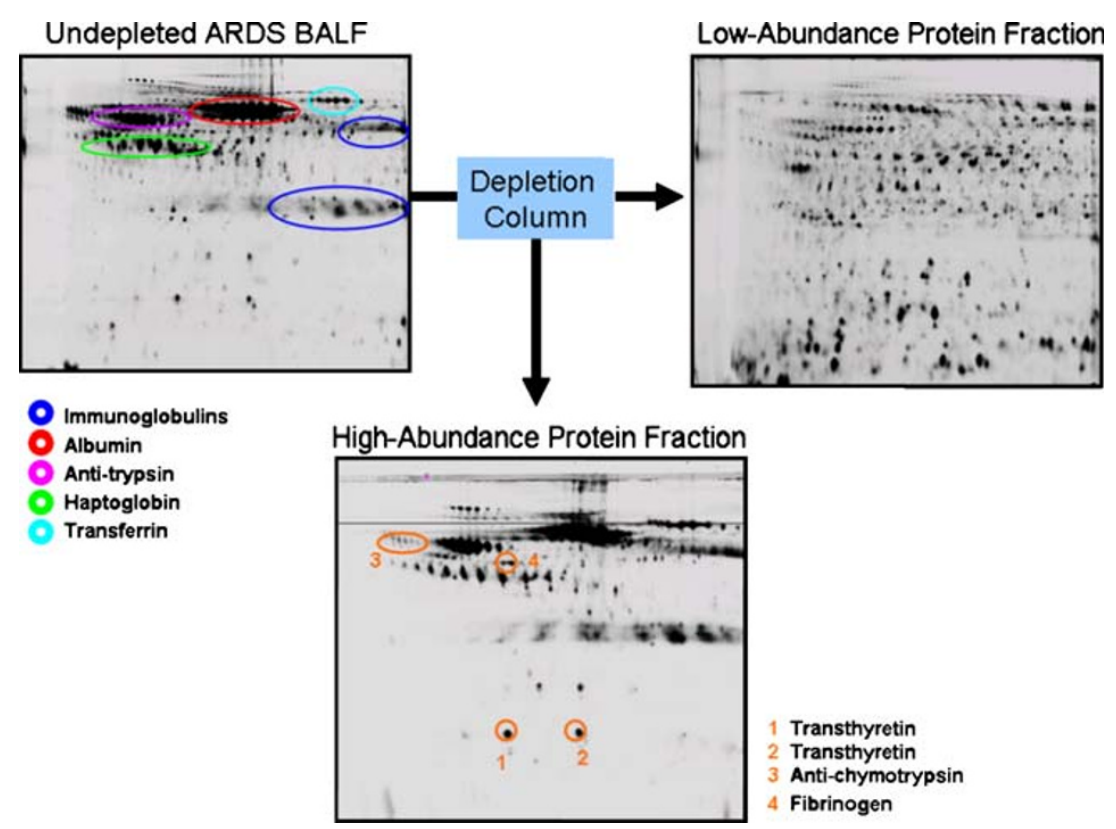

Fig. 1 High-abundance protein depletion of lung fluid samples. Twodimensional electrophoresis of undepleted ARDS lung fluid samples is limited by the presence of high-abundance serum proteins. Depletion of these proteins using an immunoaffinity column resulted

\section{Protein Identification}

Sixteen of the 17 spots that were consistently increased in the s-Cath vs mini-BAL samples were identified by mass spectrometry (Fig. 6, Table 4). One of the 17 spots could not be identified because the protein content in the spot was below the level of detection of the mass spectrometer. Several of the spots were identified as the same protein, which could be explained by protein fragmentation or posttranslation modification (Fig. 6). The 16 spots that were identified represented a total of nine different proteins (Table 4). For comparison, 15 other protein spots that were

Table 3 Summary of protein spot analyses between s-Cath and miniBAL in each subject

\begin{tabular}{lllll}
\hline $\begin{array}{l}\text { Sample } \\
\text { no. }\end{array}$ & $\begin{array}{l}\text { Number } \\
\text { of spots }\end{array}$ & $\begin{array}{l}\text { Similar } \\
(\%)\end{array}$ & $\begin{array}{l}\text { Increased in } \\
\text { s-Cath }(\%)\end{array}$ & $\begin{array}{l}\text { Decreased in } \\
\text { s-Cath }(\%)\end{array}$ \\
\hline 1 & 1,992 & 79.2 & 4.4 & 16.4 \\
2 & 2,417 & 51.1 & 24.5 & 24.4 \\
3 & 2,637 & 67.5 & 19.4 & 13.1 \\
4 & 1,951 & 67.5 & 6.9 & 25.6 \\
5 & 2,148 & 73.0 & 12.3 & 14.7 \\
6 & 1,810 & 66.4 & 15.5 & 18.1 \\
7 & 2,190 & 74.1 & 9.5 & 16.4 \\
Mean & $2,164 \pm 285$ & $68.4 \pm 8.9$ & $13.2 \pm 7.1$ & $18.4 \pm 4.8$ \\
$\quad( \pm$ SD) & & & & \\
\hline
\end{tabular}

A difference in spot abundance was defined as a twofold change in log volume ratio. The Student's $t$ test was used for comparison with a $p<$ 0.05 for statistical significance. in a threefold improvement in detection of low-abundance proteins. The high-abundance protein fraction was analyzed for the presence of low-abundance proteins. Only four protein spots representing three low-abundance proteins were found in the high-abundance fraction

similar between s-Cath and mini-BAL fluids were also excised and identified (Table 4).

\section{Discussion}

The development of non-invasive lung sampling techniques is important for both patient care and research. Before these techniques are routinely used, however, their biases and limitations must be defined. Currently, two noninvasive methods to sample the distal lung fluid (s-Cath and miniBAL) are available. Global patterns of protein expression in the lung fluid can be used as "protein fingerprints" to study the degree of similarity between the samples obtained by these collection techniques. Thus, the goal of our study was to use proteomic analysis to determine [1] whether or not the samples obtained by these methods are interchangeable and [2] whether the differences in the proteins collected by the two techniques reveal any sampling biases.

In biological fluids, the presence of high-abundance proteins often limits the detection of biologically important low-abundance proteins $[7,8]$. Thus, before performing proteomic analysis, we depleted all lung fluid samples of six high-abundance serum proteins using an immunoaffinity column. We detected an average of 2,164 spots detected per sample. Prior studies by Bowler et al. [28] using 2Delectrophoresis on s-Cath samples from patients with ARDS detected approximately 300 spots per sample. Noel-Goeris et al. [29] attempted to comprehensively 
Fig. 2 Assessment of experimental variability in proteomic analysis. Identical s-Cath samples were labeled with $\mathrm{Cy} 3$ (a) and $\mathrm{Cy} 5$ (b), and run on a single gel. A total of 2,257 spots were detected in the overlapped image. All 2,257 spots were found to be similar between the Cy3 and Cy5 images by the Decyder program (c). In the overlapped image, spots that are similar between $\mathrm{Cy} 3$ and $\mathrm{Cy} 5$ are yellow. Spots that are increased in $\mathrm{Cy} 3$ and in Cy5 are green and red, respectively
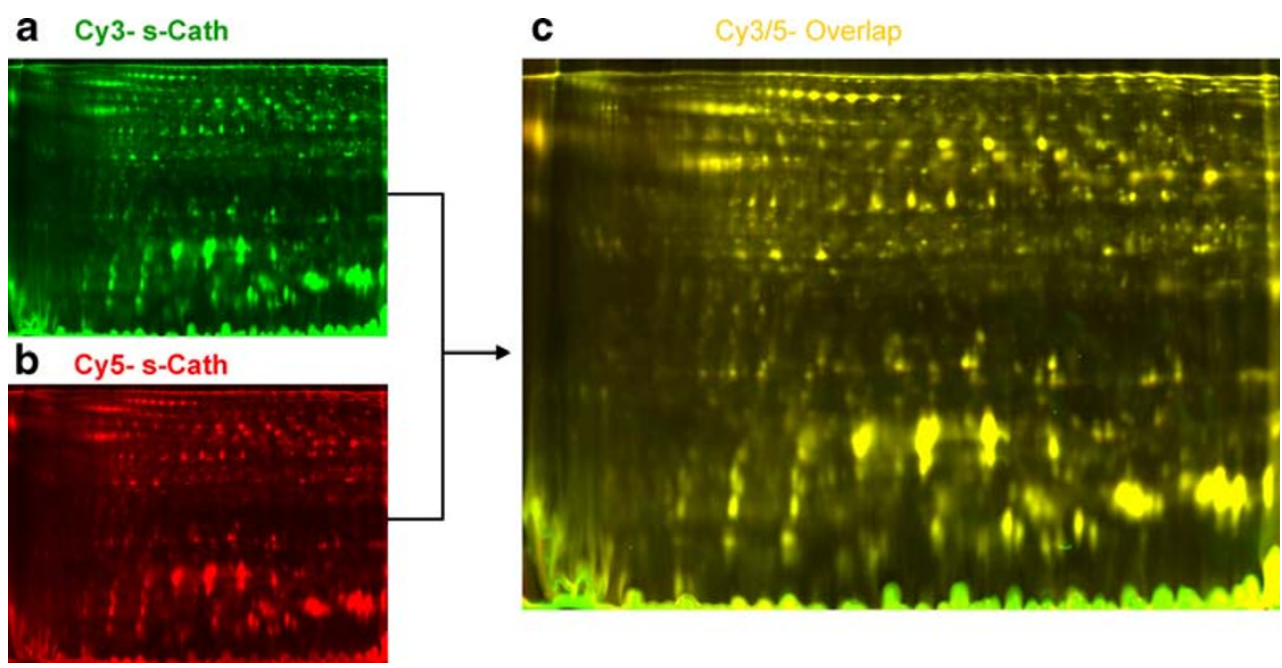

identify all proteins present in the human bronchoalveolar lavage fluid (BALF) using 2D-electrophoresis and detected over 1,200 spots using silver staining. Schnapp et al. [30], using a more sensitive mass-spectrometry-based shotgun proteomics approach, were able to identify approximately 200-650 proteins in the BALF of patients with ARDS. Our results show that, compared to earlier studies, using 2Delectrophoresis on BALF, depletion of high-abundance proteins by immunoaffinity depletion dramatically improves the resolution of low-abundance proteins. We chose to use the multiple affinity removal system (MARS) immunoaffinity column (Agilent) because previous studies have shown that MARS has a favorable sensitivity and specificity as compared with other methods of depleting high-abundance proteins [7]. We also analyzed the column fraction that contained the high-abundance proteins to determine the extent to which low-abundance proteins were also depleted as a result of protein-albumin interactions or non-specific binding to the immunoaffinity column. Only four low-abundance protein spots representing three proteins were detected in the high-abundance fraction that eluted from the column. In initial experiments, we tested the reproducibility of our depletion method (data not shown). Using 45 BALF samples from normal and ARDS subjects, we found that approximately $18 \%$ (SD, 6.1\%) of the proteins from the BALF consistently remain in the original sample after high-abundance protein depletion. Thus, the column appears to reproducibly deplete the same proportion of high-abundance proteins across many samples with low variability. We also performed high-abundance depletion on three identical aliquots of pooled BALF samples and found that greater than $85 \%$ of the spots were a Cy3-s-Cath

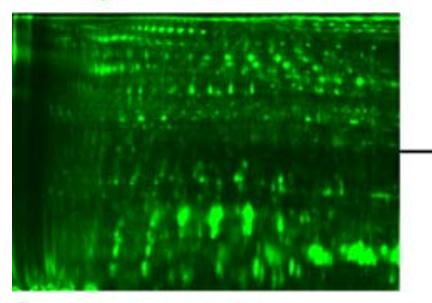

b Cy5-Mini-BAL

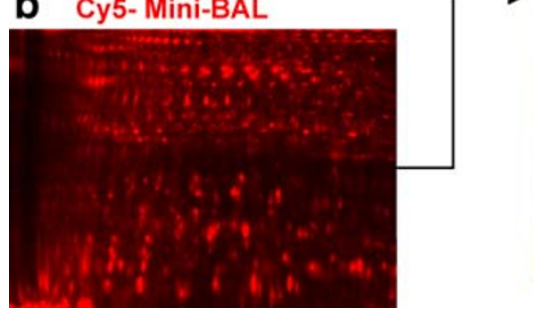

Fig. 3 Detection of differences in protein spot abundance between sCath and mini-BAL samples using 2D DIGE. The s-Cath and miniBAL samples for each patient were labeled with different fluorescent dyes and run on a single gel. The protein spots in each sample were detected separately using the unique excitation/emission character-

\section{Cy3/5-Overlap}

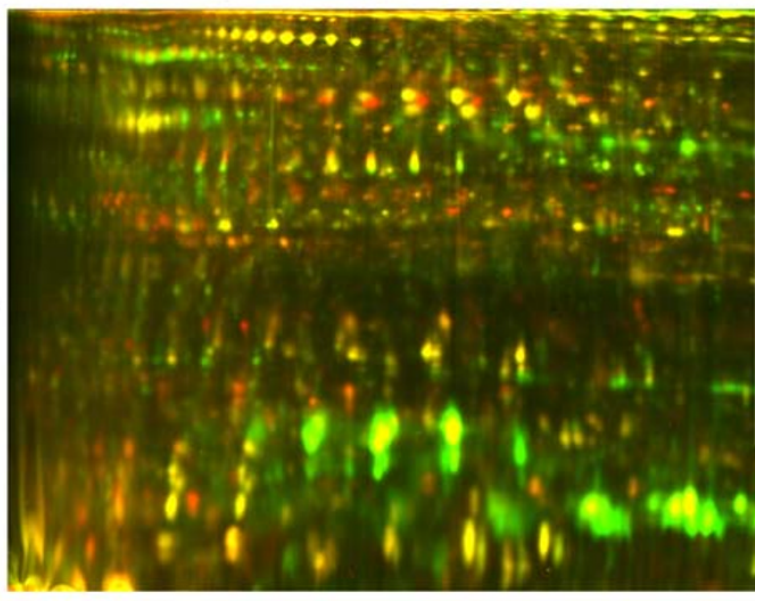

istics of the dyes (a and $\mathbf{b}$ ). The differences in protein spot abundance were quantitated using an overlapping image (c). In the overlapping image, proteins spots with similar abundances between s-Cath and mBAL are yellow. Spots that are more abundant in s-Cath are green. Spots that are more abundant in $\mathrm{mBAL}$ are red 


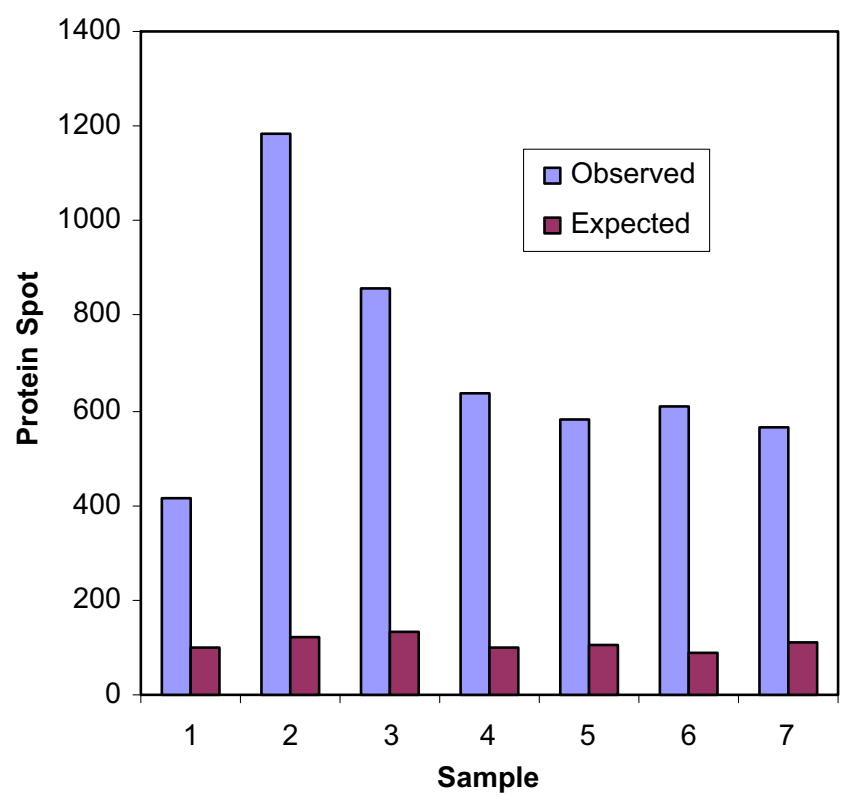

Fig. 4 Overabundance analysis of the actual and expected number of protein spots that differ between s-Cath and mini-BAL using $p<0.05$. The actual number of differentially expressed spots is greater than would be expected by chance for each of the seven subjects similar in the high-abundance fractions. This suggests that the majority of the proteins that are depleted by the column during each run are the same. In addition, two identical ARDS BALF samples were depleted separately and run on separate DIGE gels. More than $92 \%$ of the spots were similar between the two samples, suggesting that the variability in the overall methodology, including the highabundance protein depletion, is small. Our results show that, while some low-abundance proteins may be depleted along with the high-abundance proteins, these losses are minor and are outweighed by the improvement in the resolution of the other low-abundance proteins.

After the s-Cath and mini-BAL samples were depleted of high-abundance proteins, we performed proteomic analysis to compare their global protein content. The abundance of the protein spots in the s-Cath and mini-BAL samples obtained from each patient was compared to assess the interchangeability of the sampling techniques (pairwise analysis). The results of this pairwise analysis showed that there was significant variability in how similar the samples were across the seven subjects $(79 \%$ maximum similarity, $51 \%$ minimum similarity). Overall, the similarity of the samples across all patients was $68 \%$. To further study the
Fig. 5 Differentially expressed protein spots between s-Cath and mini-BAL samples. Seven hundred five spots (a and $\mathbf{b}$ ) were present in six out of seven gels and were used for groupwise comparison between the sCath and mini-BAL samples. Of these, 17 spots were differentially expressed (c and d). All 17 spots were overexpressed in the s-Cath samples. e) and f Magnified images of one of the 17 spots in $2 \mathrm{D}$ and $3 \mathrm{D}$ views. The volume of the $3 \mathrm{D}$ cone is related to protein abundance in the spot. Significant differences in spot abundance were calculated using the Student's $t$ test $(p<0.05)$

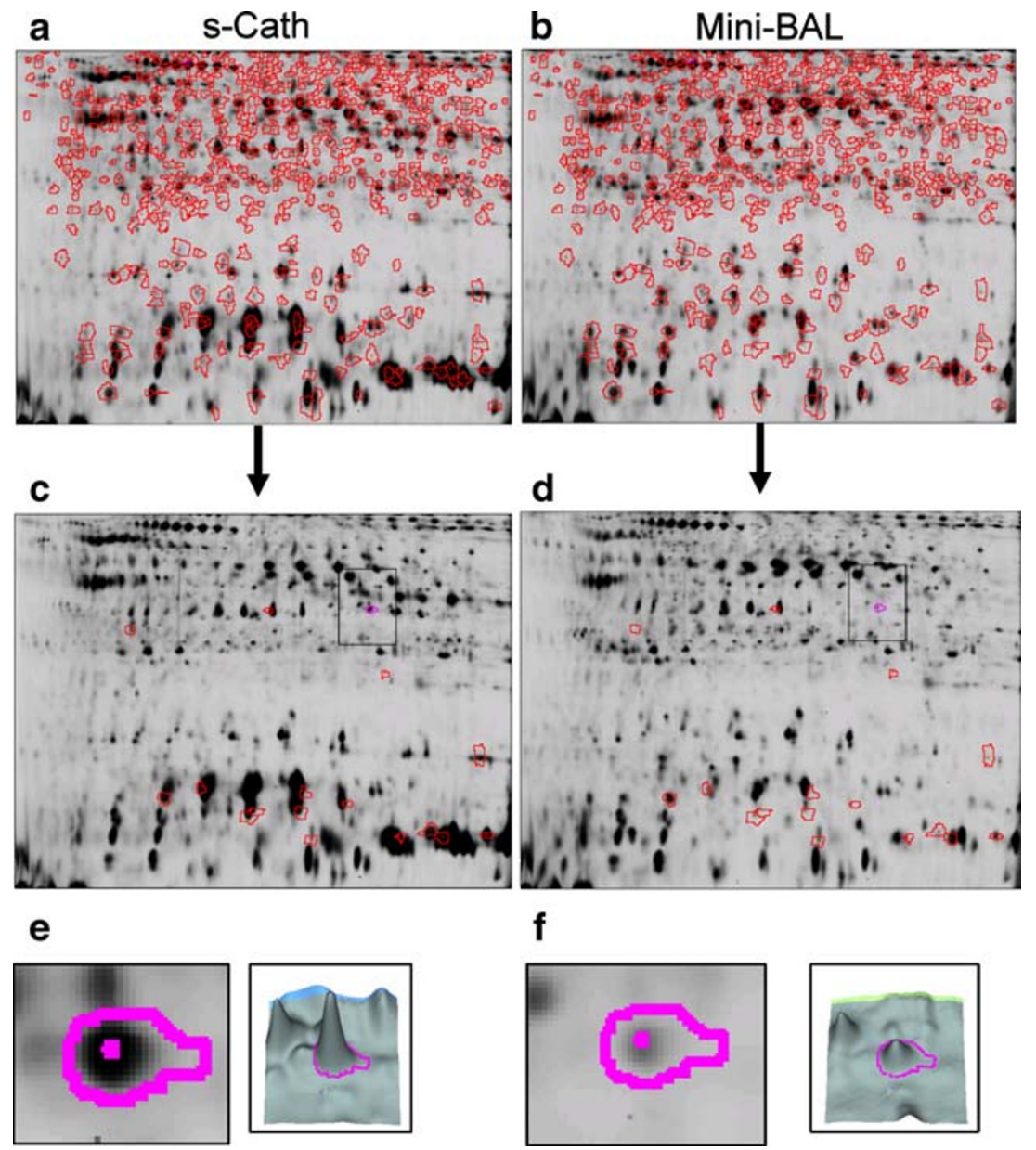




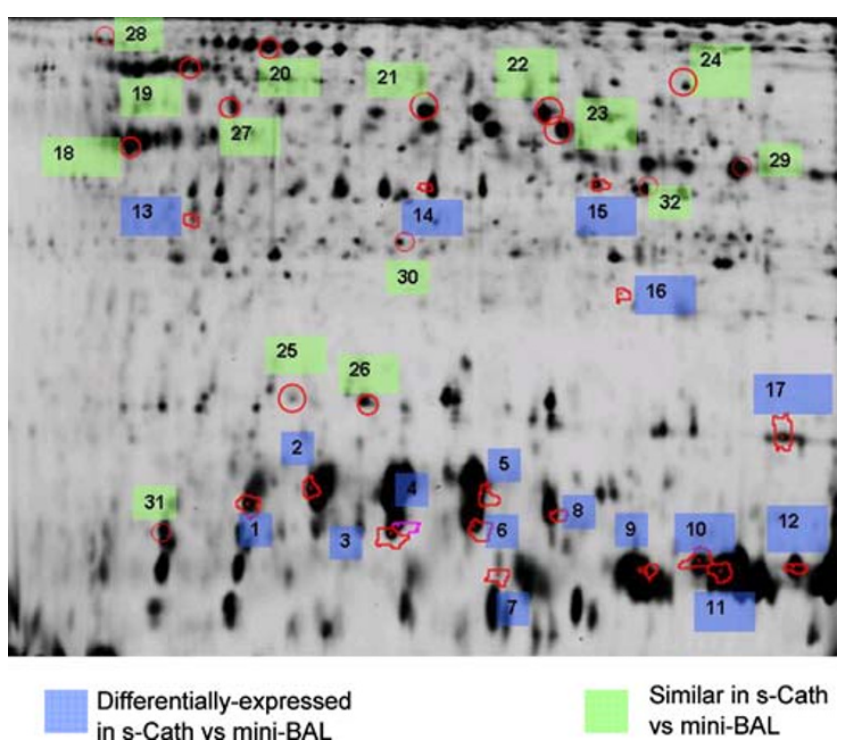

Fig. 6 The 17 spots that were differentially expressed in s-Cath vs mini-BAL were excised for mass spectrometry. In addition, ten spots that were similar between the two groups were analyzed for comparison. The protein identifications made by mass spectrometry are shown in Table 4

similarity between the samples in each patient, we calculated the expected number of differentially expressed spots, assuming that the samples were equivalent (at $p<$ 0.05 ), and compared the result to the actual number of differentially expressed spots in each patient. This overabundance analysis showed that there were many more differentially expressed spots than would be expected if the s-Cath and mini-BAL samples were interchangeable. This difference was consistent, as it was found in all seven subjects. It was also robust, with a minimum of a fourfold increase in the number of differentially expressed spots. Thus, based on the differences in the global protein content, the lung fluids obtained by the s-Cath and mini-BAL have some important differences.

To explore these differences, we performed a group-wise analysis to study the proteins that are preferentially found in either the s-Cath or mini-BAL samples. We found 17 protein spots (representing nine proteins), which were consistently different between the s-Cath and mini-BAL samples. Among these proteins, two interesting patterns emerged. First, all of the protein spots that were different between s-Cath and mini-BAL were over-expressed in the s-Cath samples. Based on this observation, we speculated that some of these proteins came from a compartment in the lung that is over-sampled by the s-Cath relative to the miniBAL. Second, four out of the nine proteins that were overexpressed in the s-Cath sample were proteins found in neutrophils (calgranulin A/B, annexin A3/A5) [9-14]. Calgranulin A and B are abundant in the cytoplasm of neutrophils and have been implicated in the regulation of inflammation [9]. Similarly, annexin A3 is expressed almost exclusively in the cytosol of myeloid cells and may have a role in vascular endothelial growth factormediated angiogenesis [10, 11]. Annexin A5 is more ubiquitously expressed but is found in leukocytes and at sites of cellular injury and apoptosis [13, 14]. Taken together, these proteins account for over $40 \%$ of the cytosolic proteins in neutrophils [9, 12]. Furthermore, this result is consistent with the cell counts in the s-Cath and mini-BAL samples, which showed a trend toward a greater percentage of neutrophils in the s-Cath compared to miniBAL samples (Table 2). These findings suggest that, as compared with the mini-BAL method, the s-Cath method tends to sample a compartment in the lung where neutrophils are more abundant.

Table 4 Protein identifications by mass spectrometry

\begin{tabular}{lll}
\hline $\begin{array}{l}\text { Spot } \\
\text { number }\end{array}$ & Protein & $\begin{array}{l}\text { Protein score } \\
\text { confidence }(\%)\end{array}$ \\
\hline
\end{tabular}

Proteins differentially expressed in s-Cath compared to mini-BAL

\begin{tabular}{|c|c|c|}
\hline 1 & Transthyretin precursor & 96 \\
\hline 2 & Calgranulin B (S100A9, MRP-14) & 100 \\
\hline 3 & Calgranulin B (S100A9, MRP-14) & 100 \\
\hline 4 & Calgranulin B (S100A9, MRP-14) & 99 \\
\hline 5 & Calgranulin B (S100A9, MRP-14) & 99 \\
\hline 6 & Calgranulin B (S100A9, MRP-14) & 100 \\
\hline 7 & No match & $\mathrm{N} / \mathrm{A}$ \\
\hline 8 & Tyrosine protein kinase ZAP-70 & 92 \\
\hline 9 & Calgranulin A (S100A8, MRP-8) & 100 \\
\hline 10 & Calgranulin A (S100A8, MRP-8) & 100 \\
\hline 11 & Calgranulin A (S100A8, MRP-8) & 100 \\
\hline 12 & Calgranulin A (S100A8, MRP-8) & 100 \\
\hline 13 & Annexin A5 & 100 \\
\hline 14 & Annexin A3 & 100 \\
\hline 15 & Squamous cell carcinoma antigen 1 & 100 \\
\hline 16 & Apolipoprotein A1 & 100 \\
\hline 17 & Hemoglobin beta chain & 100 \\
\hline \multicolumn{3}{|c|}{ Proteins similar in s-Cath and mini-BAL } \\
\hline 18 & Haptoglobin & 100 \\
\hline 19 & Alpha 1 antitrypsin & 100 \\
\hline 20 & IgA (heavy chain) & 100 \\
\hline 21 & Erythrpoietin precursor & 98 \\
\hline 22 & Leukocyte elastase inhibitor & 95 \\
\hline 23 & MAP3K12 binding inhibitory protein & 99 \\
\hline 24 & Rab GDP dissociation inhibitor & 100 \\
\hline 25 & Pescadillo homolog 1 (Pes1) & 97 \\
\hline 26 & Haptoglobin & 100 \\
\hline 27 & Fibrinogen (gamma chain) & 100 \\
\hline 28 & Alpha 1 antichymotrypsin & 100 \\
\hline 29 & Complement $\mathrm{C} 4$ & 100 \\
\hline 30 & Serum amyloid protein component & 100 \\
\hline 31 & Complement C4 & 100 \\
\hline 32 & Heat shock protein 1 & 100 \\
\hline
\end{tabular}

A cutoff of total peptides $>5$ and unique peptides $>2$ was used for protein identification. 
The observation that neutrophils are more represented in the airways than the alveolar space was first reported in studies of patients with chronic bronchitis, in which the analysis of sequential bronchoalveolar lavage aliquots showed that the neutrophil recovery was greatest in the first lavage aliquot [15]. Subsequently, Rennard et al. [16] showed that the cell content of lung fluid varies according to the segment of the lung sampled. Specifically, the bronchial airway fractions contained significantly more neutrophils and epithelial cells than the alveolar fractions [16]. These findings have been reproduced in lung diseases that cause a neutrophilic alveolitis including idiopathic pulmonary fibrosis [17], as well as other parenchymal lung diseases such as sarcoidosis [18]. Collectively, these studies suggest that lung fluid originating from the bronchial airways contains more neutrophils as compared with lung fluid from the alveoli. Thus, the overexpression of neutrophil proteins in s-Cath fluid compared to mini-BAL fluid in the same patient suggests that s-Cath samples the bronchial airways to a greater extent than does mini-BAL. This is further supported by the over-expression of a protein found exclusively in airway epithelial cells in the s-Cath samples, squamous cell carcinoma antigen-1 (SCCA-1). SCCA-1 is a serpin proteinase inhibitor that was originally isolated in human cervical squamous cell carcinomas [19]. It has since been found to be expressed in normal human bronchial epithelium and may be overexpressed in inflammatory airways diseases such as asthma [24]. Furthermore, bronchial epithelial cells were found in four out of seven s-Cath samples as compared with only two out of seven mini-BAL samples (Table 2).

Prior studies using micropuncture methods in experimental animals have shown that edema fluid sampling via the s-Cath method reflects alveolar fluid [2, 3]. However, it is well recognized that all endoluminal methods of sampling the distal lung spaces, whether it be s-Cath, mini-BAL, or bronchoscopic BAL, contain some airway contents. What is not known is how much relative airway contamination is there in each of these types of samples. Our results show that s-Cath samples the bronchial airways to a greater extent than mini-BAL. One plausible explanation for this finding relates to the method of collecting the lung fluid with each technique. The s-Cath collects lung fluid directly without the instillation of $0.9 \% \mathrm{NaCl}$. Thus, a larger proportion of the fluid sampled will come from the area immediately beyond the catheter tip, reflecting the airways. With the mini-BAL method, on the other hand, the instilled fluid is likely to flow into the alveolar spaces so that the fluid that is recovered (especially the later aliquots) is likely to reflect the alveolar contents to a greater degree.

Although the instillation of $0.9 \% \mathrm{NaCl}$ may improve the recovery of alveolar contents in lung fluid samples, one disadvantage is that the lung fluid samples become diluted compared to s-Cath samples $[25,26]$. Miller et al. [25] compared the protein concentrations of ARDS lung fluids collected by s-Cath $(N=17)$ to lung lavage samples collected by bronchoscopic BAL $(N=19)$ and found that the protein concentrations were approximately 50 times higher in the sCath samples. A comparison of the protein concentration in the lung fluid using different sampling techniques in the same patients has not been previously done [26]. Our results using s-Cath vs mini-BAL in the same patients suggest that the mini-BAL method dilutes alveolar fluids by 16-fold as compared with the s-Cath method.

To our knowledge, this is the first direct comparison of the lung fluid samples obtained by s-Cath and mini-BAL. Perkins et al. [20] compared mini-BAL to bronchoscopic BAL and found that mini-BAL samples had an increased ratio of bronchial epithelial cells to macrophages, suggesting greater airway sampling. Furthermore, mini-BAL and bronchoscopic BAL were poorly comparable as measured by Bland-Altman analysis of total protein concentration and other markers of alveolar inflammation [20]. Taken together, the study by Perkins et al. and the results of our study suggest that the lung fluid sampling technique that reflects the alveolar contents to the greatest extent is bronchoscopic BAL, followed by the mini-BAL and, finally, by the s-Cath.

This study has several limitations that must be considered in interpreting the results. First, it is difficult to assess the agreement between s-Cath and mini-BAL because the gold standard, bronchoscopic BAL, was not included in the experiment. We did not include bronchoscopy because sequentially performing mini-BAL and bronchscopic BAL in the same segment would not produce independent samples for analysis. Other statistical tools that assess agreement, such as the Bland-Altman analysis, are not compatible with the large number of variables (protein spots) in proteomic analysis. Thus, to quantitate the magnitude of difference seen between the s-Cath and mini-BAL samples, we performed an overabundance analysis.

In the overabundance analysis, the expected number of differentially expressed spots is likely to be an underestimation because our calculated value does not account for several factors that could increase the number of differentially expressed spots even if the sampling techniques are equivalent. First, the calculated value does not account for the experimental variation in sample preparation, two-dimensional electrophoresis, and spot analysis. However, our control experiment using identical s-Cath samples (Fig. 2) shows that the variability in our experimental technique is minimal and unlikely to account for the robust differences seen in the overabundance analysis. Second, because both the s-Cath and mini-BAL are performed blindly, it is possible that, although s-Cath and mini-BAL are equivalent sampling techniques, the variation in the samples in our study were the result of different segments of the lung being sampled. 
Steinberg et al. [22] compared the protein concentration of bronchoscopic BAL samples collected from the right and left lung of patients with ARDS and showed that approximately $40 \%$ of patients had a side-to-side variability of twofold or more as assessed by the Bland-Altman analysis. These results suggest that lung fluids sampled from different parts of the lung in the same patient are heterogeneous [22]. Despite the potential for heterogeneous sampling, previous investigators have examined the location of blindly inserted catheters that sample lung fluid and have found that they almost always end up in the right lower lobe [20, 23]. Perkins at al. [20] obtained chest X-rays in 4 of 21 patients in whom mini-BAL was performed and found that the catheter invariably wedges in the right bronchial tree. In addition, Pugin et al. [31] used the injection of radio-opaque contrast material in the lungs to assess the position of the mini-BAL catheter during a study of the characteristics of mini-BAL in the diagnosis of ventilator-associated pneumonia. That study also showed that the mini-BAL catheter generally wedges in the distal right lower lobe. Although no investigators have systematically studied the location of the s-Cath catheter after blind insertion, the preferential sampling of the right lower lobe likely applies to the s-Cath as well. Therefore, most of the lung fluid samples obtained by s-Cath or miniBAL were likely to have been collected from the right lower lobe. This, coupled with the consistent overabundance of differentially expressed spots in all seven subjects, argues against heterogeneous sampling as an explanation for the differences seen between the s-Cath and mini-BAL samples. Nevertheless, these confounding factors highlight the fact that the calculated number of differentially expressed spots represents an incomplete estimation of the number of spots that would be different even if the s-Cath and mini-BAL were equivalent. However, the robust differences in the actual vs expected number of spots, as well as the consistency in these differences across all subjects suggest that the samples collected by s-Cath and mini-BAL differ in some ways, although the majority of the recovered proteins were the same.

Another caveat is that this proteomic analysis probably captured only a subset of the overall lung fluid proteome. Several classes of proteins are not well represented in our proteomics dataset. Proteins with at the extremes of isoelectric points $(4>\mathrm{p} I>7)$ or molecular weight $[10>\mathrm{MW}$ $(\mathrm{kDa})>200]$ are not likely to show on the analytical portion of the 2D gel. Furthermore, extremely low-abundance proteins, such as chemokines and cytokines, are typically below the level of detection for the DIGE methodology. Finally, proteins that are membrane-bound or hydrophobic also are less likely to be detected by the DIGE methodology.

Despite these considerations, this proteomic analysis using the DIGE method leads to a testable hypothesis that can be explored in larger numbers of patients using surrogate markers of airway sampling (such as the number of bronchial epithelial cells) to confirm the results.

\section{Conclusions}

In summary, we have shown using a proteomic analysis that, while the results of lung sampling by the s-Cath and mini-BAL methods are generally similar, there are some important differences. Analysis of the global protein expression in these lung fluid samples suggests that the differences occur because the s-Cath samples the bronchial airways to a greater extent than the mini-BAL method.

\section{References}

1. Matthay MA, Wiener-Kronish JP. Intact epithelial barrier function is critical for the resolution of alveolar edema in humans. Am Rev Respir Dis 1990;142:1250-7.

2. Berthiaume Y, Broaddus VC, Gropper MA, et al. Alveolar liquid and protein clearance from normal dog lungs. J Appl Physiol 1988;65:585-93.

3. Verghese GM, Ware LB, Matthay BA, et al. Alveolar epithelial fluid transport and the resolution of clinically severe hydrostatic pulmonary edema. J Appl Physiol 1999;87:1301-12.

4. Bernard GR, Artigas A, Brigham KL, et al. The AmericanEuropean Consensus Conference on ARDS. Definitions, mechanisms, relevant outcomes and clinical trial coordination. Am J Respir Crit Care Med 1994;149:818-24.

5. Alban A, David SO, Bjorkesten L, et al. A novel experimental design for comparative two-dimensional gel analysis: two-dimensional difference gel electrophoresis incorporating a pooled internal standard. Proteomics 2003;3:36-44.

6. Ware L, Matthay M. The acute respiratory distress syndrome. N Engl J Med. 2000;342:1334-49.

7. Bjorhall K, Miliotis T, Davidsson P. Comparison of different depletion strategies for improved resolution in proteomic analysis of human serum samples. Proteomics 2005;5:307-17.

8. Echan LA, Tang HY, Ali-Khan N, et al. Depletion of multiple high-abundance proteins improves protein profiling capacities of human serum and plasma. Proteomics. 2005;5:3292-303.

9. Nacken W, Roth J, Sorg C, et al. S100A9/S100A8: myeloid representatives of the $\mathrm{S} 100$ protein family as prominent players in innate immunity. Microsc Res Tech 2003;60:569-80.

10. Tait JF, Frankenberry DA, Miao CH, et al. Chromosomal localization of the human annexin III (ANX3) gene. Genomics 1991;10:441-8.

11. Park JE, Lee DH, Lee JA, et al. Annexin A3 is a potential angiogenic mediator. Biochem Biophys Res Commun 2005;337:1283-7.

12. Coméra C, Rothhut B, Cavadore JC, et al. Further characterization of four lipocortins from human peripheral blood mononuclear cells. J Cell Biochem 1989;40:361-70.

13. Carcedo MT, Iglesias JM, Bances P, et al. Functional analysis of the human annexin A5 gene promoter: downstream DNA element and upstream long terminal repeat regulate transcription. Biochem J. 2001;356:571-9.

14. Boersma HH, Kietselaer BL, Stolk LM, et al. Past, present, and future of annexin A5: from protein discovery to clinical applications. J Nucl Med. 2005;46:2035-50.

15. Martin TR, Raghu G, Maunder RH, et al. The effects of chronic bronchitis and chronic airflow obstruction on lung cell popula- 
tions recovered by bronchoalveolar lavage. Am Rev Respir Dis 1985;132:254-60.

16. Rennard SI, Ghafouri MO, Thompson AB, et al. Fractional processing of sequential bronchoalveolar lavage to separate bronchial and alveolar samples. Am Rev Respir Dis 1990;141:208-17.

17. Yasuoka S, Nakayama T, Kawano T, et al. Comparison of cell profiles of bronchial and bronchoalveolar lavage fluids between normal subjects and patients with idiopathic pulmonary fibrosis. Tohoku J Exp Med 1985;146(1):33-45.

18. Winterbauer RH, Wu R, Springmeyer SC. Fractional analysis of the 120-ml bronchoalveolar lavage: determination of the best specimen for the diagnosis of sarcoidosis. Chest 1993;104:4344-51.

19. Kato H. Expression and function of squamous carcinoma cell antigen. Anticancer Res 1996;16:2149-53.

20. Perkins GD, Chatterjie S, McAuley DF, et al. Role of nonbronchoscopic lavage for investigating alveolar inflammation and permeability in acute respiratory distress syndrome. Crit Care Med 2005;33:57-64.

21. Perkins GD, Chatterjie S, Giles S, et al. Safety and tolerability of nonbronchoscopic lavage in ARDS. Chest 2005;127:1358-63.

22. Steinberg KP, Ruzinski J, Caldwell E, et al. The heterogeneity of BAL cells and protein in patients at risk for and with ARDS (Abstract). Chest 1999;116:98S.

23. Heaney LG, Stevenson EC, Turner G, et al. Investigating paediatric airways by non-bronchoscopic lavage: normal cellular data. Clin Exp Allergy. 1996;26:799-806.
24. Yuyama N, Davies DE, Akaiwa M, et al. Analysis of novel diseaserelated genes in bronchial asthma. Cytokine 2002;19:287-96.

25. Miller EJ, Nagao S, Griffith D, et al. Elevated levels of NAP-1/ interleukin-8 are present in the airspaces of patients with the adult respiratory distress syndrome and are associated with increased mortality. Am Rev Respir Dis 1992;146:427-32.

26. Martin TR, Goodman RB. The role of chemokines in the pathophysiology of the acute respiratory distress syndrome (ARDS). In: Hebert CA, editor. Chemokines in disease: biology and clinical research. 1st ed. Totowa: Humana; 1999. p. 81-110.

27. Shevchenko A, WIlm M, Vorm O, et al. Mass spectrometric sequencing of proteins from silver-stained polyacrylamide gels. Anal Chem 1996;68:850-8.

28. Bowler RP, Duda B, Chan E, et al. Proteomic analysis of pulmonary edema fluid and plasma in patients with acute lung injury. Am J Physiol Lung Cell Mol Physiol 2004;286:L1095-104.

29. Noel-Georis I, Bernard A, Falmagne P, Wattiez R. Database of bronchoalveolar lavage proteins. J Chromatogr B 2002;771:221-36.

30. Schnapp LM, Donohoe S, Chen J, et al. Mining the acute respiratory distress syndrome proteome: Identification of the insulin-like growth factor (IGF)/IGF-binding protein-3 pathway in acute lung injury. Am J Pathol 2006;169:86-95.

31. Pugin J, Auckenthaler R, Mili N, et al. Diagnosis of ventilatorassociated pneumonia by bacteriologic analysis of bronchoscopic and nonbronchoscopic "blind" bronchoalveolar lavage fluid. Am Rev Respir Dis 1991;143:1121-29. 